\title{
Dietary inclusion of oil palm fronds does not change n-6 nor n-3 content of lamb tissue
}

\begin{abstract}
Inclusion of oil palm fronds (OPF) in ruminant diets was previously reported to increase the tissue content of unsaturated fatty acids (UFAs) and reduce rumen biohydrogenation, which was in contrast with our former in vitro study. Thus, a small scale in vivo study was performed to assess the effects of different inclusion levels of OPF on UFA content of intramuscular fat (longissimus dorsi, biceps femoris) and adipose tissues (subcutaneous, perirenal). Sixteen lambs were divided into 4 treatment groups, with dietary inclusion levels of OPF of $0,100,200$ and $300 \mathrm{~g} / \mathrm{kg} \mathrm{DM}$, respectively. Further, the diet contained a commercial sheep/goat pellet. Diets were supplemented with soybean oil (40 g/kg DM), which was equally mixed with the whole ration. Hence, the diet particularly supplied linoleic acid (LA; 563-569 g/kg total fatty acid (FA) intake). Due to selective refusal of the OPF, actual proportion of OPF in the ingested feed was lower compared with the offered diet, i.e. 44, 137 and $170 \mathrm{~g} / \mathrm{kg}$ DM. The total fat intake also differed among the treatments due to differences in feed intake. After 12 weeks on the experimental diets, the lambs were slaughtered and both intramuscular fat and adipose tissues were collected for FA analysis. No differences were observed in total FA content, proportion of C18:2 n-6 and UFA/SFA ratio in OPF inclusion groups for both intramuscular fat and adipose tissues. Hence, OPF does not seem to relatively modify UFA content of adipose or intramuscular fat.
\end{abstract}

Keyword: Oil palm fronds; Muscle; Adipose tissue; Unsaturated fatty acid; Biohydrogenation 